This article was published in the following Dove Press journal:

Cancer Management and Research

\section{Rui Li (iD ${ }^{1, *}$ \\ Zhen Sun ${ }^{1, *}$ \\ Shibo Song ${ }^{2}$ \\ Xiuwen $\mathrm{He}^{1}$ \\ Xiaolei Shi ${ }^{1}$ \\ Zhe $\mathrm{Li}^{\prime}$ \\ Jinghai Song $\mathbb{D}^{\prime}$}

'Department of General Surgery, Beijing Hospital, Peking University Fifth School of Clinical Medicine, National Center of Gerontology; Institute of Geriatric Medicine, Chinese Academy of Medical Science, Beijing, 100730, People's Republic of China; ${ }^{2}$ Department of Colorectal Surgery, Tianjin Union Medical Center, Tianjin 300 I2I, People's Republic of China

*These authors contributed equally to this work
Correspondence: Jinghai Song

Department of General Surgery, Beijing Hospital, Peking University Fifth School of Clinical Medicine, National Center of Gerontology; Institute of Geriatric Medicine, Chinese Academy of Medical Science, No. I, Dahua Road, Beijing 100730, People's Republic of China

Tel +86-10-85I36262

Fax +86-10-65132969

Email jhaisong2003@I63.com
Objective: The neutrophil-to-albumin ratio (NAR) and fibrinogen are significantly related to tumor progression. The present study evaluated the prognostic impact of the NAR plus fibrinogen concentration in gastrointestinal stromal tumor (GIST) cases.

Methods: The baseline characteristics, postoperative NAR, and fibrinogen concentrations were retrospectively analyzed for 229 Chinese patients who underwent radical gastrectomy for GIST. Receiver operating characteristic (ROC) curves were applied to estimate the optimal critical points for NAR and fibrinogen. Cox regression analysis was applied to determine significant prognostic variables.

Results: Multivariate analyses revealed that poor recurrence-free survival was associated with elevated values for fibrinogen (hazard ratio [HR]: 5.015, 95\% confidence interval [CI]: 1.993-12.619, $\mathrm{P}=0.001$ ) and NAR (HR: 4.669, 95\% CI: 1.776-12.273, $\mathrm{P}=0.002$ ). Combining fibrinogen and the NAR into the NARFIB score provided an area under the ROC curve of 0.833 , which was greater than the areas for NAR $(0.708)$ or fibrinogen $(0.778)$. When the NAR and fibrinogen were replaced by the NARFIB score in the multivariate analysis, the independent prognosticators were tumor site (HR: 2.927, 95\% CI: 1.417-6.045, $\mathrm{P}=0.004)$, mitotic index (HR: 2.661, 95\% CI: 1.110-6.380, $\mathrm{P}=0.028)$, and the NARFIB score (HR: 14.116, 95\% CI: 3.243-61.443, $\mathrm{P}<0.001$ ). The NARFIB score retained its prognostic significance in various subgroup analyses and was significantly related to gender, surgical approach, tumor size, mitosis, tumor site, risk classification, and recurrence.

Conclusion: These results suggest that the NARFIB score may help guide prognostication and risk stratification for GIST, which might benefit from targeted therapy.

Keywords: neutrophil-to-albumin ratio, fibrinogen, prognosis, gastrointestinal stromal tumors

\section{Introduction}

In the digestive tract, the most common mesenchymal tumors are gastrointestinal stromal tumors (GISTs), which have an annual incidence rate of 10-15 cases/ $1,000,000$ population. The GIST tumor types vary from benign tumors to fatal sarcomas, and the optimal treatment for GIST is curative resection. ${ }^{1}$ The prognosis of GIST has improved after the introduction of imatinib as targeted therapy, although relapse after radical resection remains common, especially among high-risk patients, and $<50 \%$ are free from recurrence at 5 years after surgery. ${ }^{2}$ Moreover, the median survival 
time is $<2$ years after relapse. ${ }^{1,2}$ The modified National Institutes of Health (mNIH) category is the most common risk classification for GIST, which aims to predict and stratify the risk of GIST relapse according to mitotic count, tumor location, tumor size, and rupture. ${ }^{3}$ However, these parameters require pathological evaluation of tumor specimens and it would be useful to have non-invasive and effective methods for screening and prognostication among moderateand high-risk cases of GIST, which might benefit from targeted therapy.

Various studies have indicated that tumorigenesis and tumor progression are related to systemic inflammation, malnutrition, immune status, and hypercoagulability. ${ }^{4-6}$ For example, the progression of various tumors is related to the neutrophil-to-albumin (NAR) ratio and fibrinogen concentration. Furthermore, the NAR is an independent prognosticator of pathological complete remission post-chemotherapy for rectal cancer, as well as a predictor of survival among patients receiving palliative treatment for pancreatic cancer. ${ }^{7,8}$ Fibrinogen concentrations also have prognostic value for hepatocellular carcinoma, ${ }^{9}$ colorectal cancer, ${ }^{10}$ and GIST. ${ }^{11}$ Therefore, we speculated that a score that combined the NAR and fibrinogen concentration (the NARFIB score) might be useful for prognostication in cases of GIST, and we retrospectively tested this hypothesis among cases who had received curative surgery for GIST.

\section{Patients and Methods}

\section{Patients}

We retrospectively identified 229 cases that had received curative surgery for pathologically confirmed GIST at our center between November 2003 and August 2018, and evaluated their clinicopathological data. The inclusion criteria were (1) patients with complete clinicopathological data and follow-up records, (2) age of $\geq 18$ years, and (3) patients with no neoadjuvant therapy before surgery. The exclusion criteria were (1) a history of anticoagulation or albumin transfusions within 3 months before the surgery, (2) patients with active inflammation, (3) patients with malignant tumors, (4) patients with hematological diseases or connective tissue diseases, and (5) patients with R1-R2 resection. The Beijing Hospital Medical Ethics Committee approved the study.

\section{Data Collection}

The clinicopathological facts included age, gender, body mass index (BMI), surgical approach, operative time, tumor site, tumor rupture, tumor size, mitosis, mNIH category, neutrophil count, hemoglobin concentration, albumin concentration, fibrinogen concentration, adjuvant imatinib treatment. The data regarding neutrophil count, hemoglobin concentration, albumin concentration, and fibrinogen concentration were all collected $<7$ days before the operation. The NAR was calculated as the neutrophil count $\left(10^{9} / \mathrm{L}\right)$ divided by the albumin level $(\mathrm{g} / \mathrm{L})$.

\section{Follow-Up}

The patients underwent follow-up evaluations every 6 months for 3 years post-operation, and then once a year after 3 years. The most recent follow-up was performed on September 20, 2019. The follow-up evaluations consisted of magnetic resonance imaging or computed tomography of the chest, pelvis, and abdomen, as well as endoscopy and bone marrow scans if necessary. Relapse-free survival (RFS) refers to the time from operation to tumor recurrence.

\section{Calculating the NARFIB Score}

The receiver operating characteristic (ROC) curves revealed optimal critical points of 0.086 for the NAR and $3.57 \mathrm{~g} / \mathrm{L}$ for fibrinogen concentration, based on the areas under the curves (AUCs) and 95\% confidence intervals (CIs). Combining NAR and FIB, we categorized patients into three "NARFIB" groups: NARFIB0 $=$ NAR $<0.086$ and fibrinogen $<3.57 \mathrm{~g} / \mathrm{L}, \mathrm{NARFIB} 1=$ either $\mathrm{NAR} \geq 0.13$ or fibrinogen $\geq 3.57$ $\mathrm{g} / \mathrm{L}, \mathrm{NARCA} 2=\mathrm{NAR} \geq 0.13$ and fibrinogen $\geq 3.57 \mathrm{~g} / \mathrm{L}$.

\section{Statistical Analysis}

SPSS software 22.0 was applied for all statistical analyses. The ROC curves were applied to evaluate the prognostic role of NAR, fibrinogen concentration, and the NARFIB score, with optimal critical points determined using the estimated Youden index (sensitivity + specificity-1). The chi-squared test or Fisher's exact test was applied to assess the relationships between clinicopathological variables and the NARFIB score. The Kaplan-Meier method was conducted to generate survival curves and the Log rank test was applied to compare. The prognostic value of various clinicopathological variables was evaluated by Cox regression analysis. $\mathrm{P}<0.05$ was considered statistically significant.

\section{Results}

\section{Clinicopathological Factors}

Table 1 shows the 229 cases' clinicopathological characteristics. The median age was 62 years (range: 18-83 
Table I Correlations Between the NARFIB Score and Clinicopathological Characteristics

\begin{tabular}{|c|c|c|c|c|c|}
\hline \multirow[t]{2}{*}{ Factor } & \multicolumn{2}{|l|}{ Total } & \multicolumn{2}{|l|}{ NARFIB } & \multirow[t]{2}{*}{$P$ value } \\
\hline & $(n=229)$ & $0(n=120)$ & $I(n=69)$ & $2(n=40)$ & \\
\hline \multicolumn{6}{|l|}{ Age } \\
\hline$<60$ years & 96 (4I.9\%) & $56(46.7 \%)$ & 27 (39.1\%) & $13(32.5 \%)$ & 0.248 \\
\hline$\geq 60$ years & $133(58.1 \%)$ & $64(53.3 \%)$ & $42(60.9 \%)$ & $27(67.5 \%)$ & \\
\hline \multicolumn{6}{|l|}{ Gender } \\
\hline Male & I 25 (54.6\%) & 56 (46.7\%) & 41 (59.4\%) & $28(70.0 \%)$ & 0.023 \\
\hline Female & 104 (45.4\%) & $64(53.3 \%)$ & $28(40.6 \%)$ & $12(30.0 \%)$ & \\
\hline \multicolumn{6}{|l|}{ Body mass index } \\
\hline$<24 \mathrm{~kg} / \mathrm{m}^{2}$ & 117 (5I.1\%) & 58 (48.3\%) & $38(55.1 \%)$ & $2 \mathrm{I}(52.5 \%)$ & 0.659 \\
\hline$\geq 24 \mathrm{~kg} / \mathrm{m}^{2}$ & 112 (48.9\%) & $62(51.7 \%)$ & 31 (44.9\%) & $19(47.5 \%)$ & \\
\hline \multicolumn{6}{|l|}{ Approach } \\
\hline Laparotomy & |3| (57.2\%) & 58 (48.3\%) & 45 (65.2\%) & $28(70 \%)$ & 0.015 \\
\hline Laparoscopic surgery & 98 (42.8\%) & $62(51.7 \%)$ & 24 (34.8\%) & $12(30 \%)$ & \\
\hline \multicolumn{6}{|l|}{ Operative time } \\
\hline$<180 \min$ & $160(69.9 \%)$ & $83(69.2 \%)$ & $53(76.8 \%)$ & $24(60.0 \%)$ & 0.177 \\
\hline$\geq 180 \mathrm{~min}$ & $69(30.1 \%)$ & $37(30.8 \%)$ & $16(23.2 \%)$ & $16(40.0 \%)$ & \\
\hline \multicolumn{6}{|l|}{ Tumor site } \\
\hline Gastric & 159 (69.4\%) & 89 (74.2\%) & 49 (71.0\%) & $2 \mathrm{I}(52.5 \%)$ & 0.034 \\
\hline Extra-gastric & 70 (30.6\%) & 31 (25.8\%) & $20(29.0 \%)$ & $19(47.5 \%)$ & \\
\hline \multicolumn{6}{|l|}{ Tumor rupture } \\
\hline No & 227 (99.1\%) & $120(100.0 \%)$ & 67 (97.1\%) & $40(100.0 \%)$ & 0.120 \\
\hline Yes & $2(0.9 \%)$ & $0(0.0 \%)$ & $2(2.9 \%)$ & $0(0.0 \%)$ & \\
\hline \multicolumn{6}{|l|}{ Tumor size } \\
\hline$<5 \mathrm{~cm}$ & 135 (59\%) & 90 (75.0\%) & 33 (47.8\%) & $12(30.0 \%)$ & $<0.001$ \\
\hline$\geq 5 \mathrm{~cm}$ & 94 (4I\%) & $30(25.0 \%)$ & 36 (52.2\%) & $28(70.0 \%)$ & \\
\hline \multicolumn{6}{|l|}{ Mitosis } \\
\hline$<5 / 50 \mathrm{HPFs}$ & |3| (57.2\%) & $84(70.0 \%)$ & $34(49.3 \%)$ & $13(32.5 \%)$ & $<0.001$ \\
\hline$\geq 5 / 50$ HPFs & 98 (42.8\%) & $36(30.0 \%)$ & $35(50.7 \%)$ & $27(67.5 \%)$ & \\
\hline \multicolumn{6}{|l|}{ mNIH category } \\
\hline Very low/low & 115 (50.2\%) & 75 (62.5\%) & $28(40.6 \%)$ & $12(30.0 \%)$ & $<0.001$ \\
\hline Intermediate/high & II 4 (49.8\%) & 45 (37.5\%) & $4 \mathrm{I}(59.4 \%)$ & $28(70.0 \%)$ & \\
\hline \multicolumn{6}{|l|}{ Hemoglobin } \\
\hline$<125 \mathrm{~g} / \mathrm{L}$ & 91 (39.7\%) & 37 (30.8\%) & 38 (55.1\%) & $16(40.0 \%)$ & 0.005 \\
\hline$\geq 125 \mathrm{~g} / \mathrm{L}$ & $138(60.3 \%)$ & $83(69.2 \%)$ & 31 (44.9\%) & $24(60.0 \%)$ & \\
\hline \multicolumn{6}{|l|}{ Adjuvant imatinib } \\
\hline No & I 78 (77.7\%) & $100(83.3 \%)$ & $49(71.0 \%)$ & $29(72.5 \%)$ & 0.100 \\
\hline Yes & 51 (22.3\%) & $20(16.7 \%)$ & 20 (29.0\%) & II (27.5\%) & \\
\hline
\end{tabular}

Abbreviations: NARFIB, score combining the neutrophil-to-albumin ratio and fibrinogen; HPFs, high-powered fields; mNIH, modified National Institutes of Health classification.

years) and the group included 125 men (54.6\%) and 104 women (45.4\%). The median BMI was $23.9 \mathrm{~kg} / \mathrm{m}^{2}$ (range: $\left.16.4-36.6 \mathrm{~kg} / \mathrm{m}^{2}\right)$. The surgical approaches were categorized as laparotomy for 131 patients $(57.2 \%)$ and laparoscopic surgery for 98 patients $(42.8 \%)$. Tumor location was categorized as in the stomach for 159 patients $(69.4 \%)$ or in other digestive tract locations for 70 patients $(30.6 \%)$. Tumor rupture was only confirmed for 2 patients $(0.9 \%)$. The median tumor size was $4.0 \mathrm{~cm}$ (range: $0.3-22.0 \mathrm{~cm}$ ). Based on the $\mathrm{mNIH}$ classification 
system, very low risk was judged for 42 patients (18.3\%), low risk was judged for 73 patients (31.9\%), intermediate risk was judged for 40 patients (17.5\%), and high risk was judged for 74 patients (32.3\%). The median hemoglobin concentration was $128 \mathrm{~g} / \mathrm{L}$ (range: $38-168 \mathrm{~g} / \mathrm{L}$ ), the median NAR was 0.072 (range: $0.030-0.344$ ), and the median fibrinogen concentration was $3.15 \mathrm{~g} / \mathrm{L}$ (range: $1.22-10.36$ $\mathrm{g} / \mathrm{L}$ ). Among the 114 patients of the moderate/high-risk group, $51(44.7 \%)$ patients received adjuvant imatinib treatment following surgery.

\section{ROC Analysis}

According to the ROC curves (Figure 1), the optimal critical values were defined as 0.086 for the NAR (AUC: 0.708, 95\% CI: $0.605-0.811 ; \mathrm{p}<0.001)$ and $3.57 \mathrm{~g} / \mathrm{L}$ for fibrinogen concentrations (AUC: 0.778, 95\% CI: 0.690-0.867; $\mathrm{p}<0.001)$. Using these critical values, the AUC for the NARFIB score was 0.833 (95\% CI: 0.605-0.811; $\mathrm{p}<0.001$ ), which was superior to the AUC for NAR or fibrinogen concentration alone.

\section{Survival Analysis}

The median RFS was 38.5 months (range: 2.9-166.0 months), and one-year, three-year and five-year RFS rates were $96.8 \%, 88.0 \%$, and $81.7 \%$, respectively. The patients were grouped according to a NARFIB score of 0 points (120 patients, 52.4\%), 1 point (69 patients, 30.1\%), or 2 points (40 patients, $17.5 \%$ ). The Kaplan-Meier survival curves shown that higher NARFIB scores were

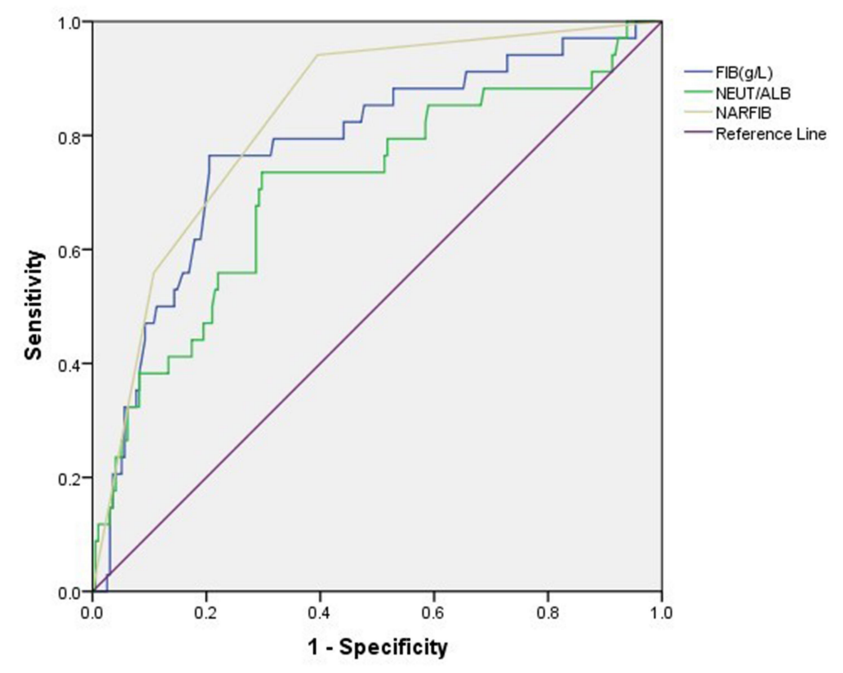

Figure I Optimal critical points for the neutrophil-to-albumin ratio, fibrinogen, and the NARFIB score were determined using receiver operating characteristic curves. Abbreviations: FIB, fibrinogen; NEUT/ALB, neutrophil/albumin; NARFIB, score combining the neutrophil-to-albumin ratio and fibrinogen. significantly related to poorer RFS ( $p<0.001$, Figure 2 ). One-year, three-year and five-year RFS rates for the NARFIB 0 group were $100.0 \%, 96.8 \%$, and $81.7 \%$, respectively, for the NARFIB 1 group were 96.9\%, $85.0 \%$, and $82.4 \%$, and for the NARFIB 2 group were $86.8 \%, 62.2 \%$, and $31.7 \%$.

The univariate analyses (Table 2) revealed that RFS was significantly associated with surgical approach (hazard ratio [HR]: $0.237,95 \%$ CI: $0.091-0.613, p=0.003)$, tumor site (HR: $3.417,95 \%$ CI: $1.735-6.729, \mathrm{p}<0.001$ ), tumor rupture (HR: 31.822, 95\% CI: 3.798-266.649, $\mathrm{p}=0.001$ ), larger tumor size (HR: $3.935,95 \%$ CI: $1.880-8.237, \mathrm{p}<0.001$ ), higher mitotic index (HR: 4.527, 95\% CI: 2.109-9.718, $\mathrm{p}<0.001$ ), and higher NARFIB score (HR: 20.272, 95\% CI: 4.856-84.623, $\mathrm{p}<0.001)$. The multivariate analyses suggested that poor RFS among GIST patients was independently related with higher mitotic index (HR: 2.737, 95\% CI: $1.095-6.841, \mathrm{p}=0.031$ ), tumor rupture (HR: 21.305, 95\% CI: 2.125-213.603, $\mathrm{p}=0.009$ ), tumor site (HR: 1.974 , 95\% CI: 0.912-4.272, $\mathrm{p}=0.084$ ), the NAR (HR: $4.669,95 \%$ CI: 1.776-12.273, $\mathrm{p}=0.002$ ), and fibrinogen (HR: 5.015, 95\% CI: $1.993-12.619, \mathrm{p}=0.001)$, although tumor size and surgical approach were not independent prognosticators. When the NAR as well as fibrinogen were replaced by the NARFIB score in the multivariate analysis, the independent prognosticators were tumor site (HR: $2.927,95 \%$ CI: 1 .417-6.045, $\mathrm{P}=0.004$ ), mitotic index (HR: $2.661,95 \% \mathrm{CI}$ : 1.110-6.380, $\mathrm{P}=0.028$ ), and the NARFIB score (HR: 14.116, 95\% CI: 3.243-61.443, $\mathrm{P}<0.001)$. Subgroup

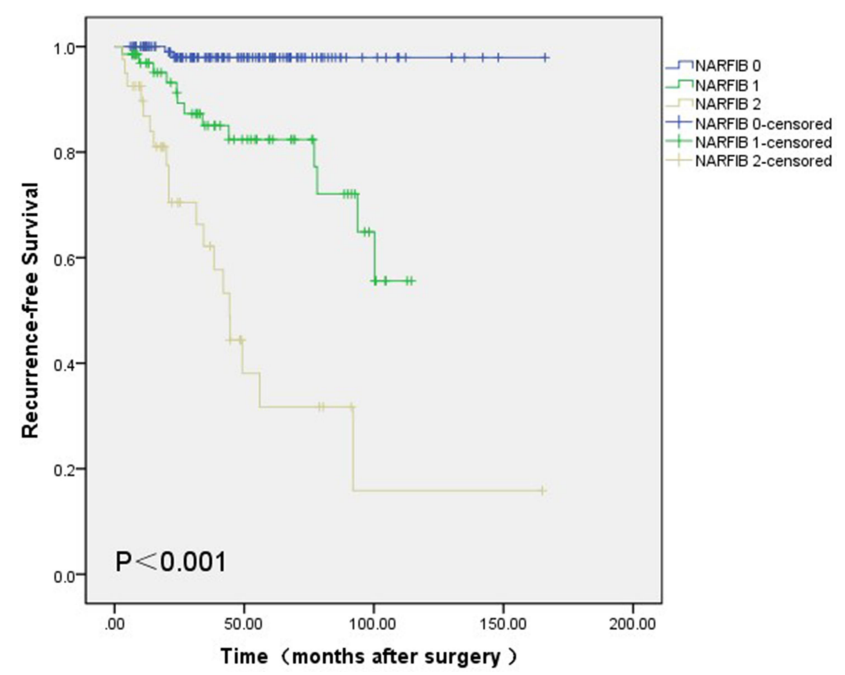

Figure 2 Kaplan-Meier curves for recurrence-free survival according to the NARFIB score.

Abbreviation: NARFIB, score combining the neutrophil-to-albumin ratio and fibrinogen. 
Table 2 Univariate and Multivariate Analyses of Clinicopathological Variables That Were Related to Recurrence-Free Survival

\begin{tabular}{|c|c|c|c|c|c|c|}
\hline \multirow[t]{2}{*}{ Variables } & \multicolumn{2}{|l|}{ Univariate Analysis } & \multicolumn{2}{|c|}{ Multivariate Analysis(I) } & \multicolumn{2}{|c|}{ Multivariate Analysis(2) } \\
\hline & HR (95\% Cl) & $P$ & HR (95\% Cl) & $P$ & HR (95\% Cl) & $P$ \\
\hline \multicolumn{7}{|l|}{ Age } \\
\hline$<60$ years & 1 & & & & & \\
\hline$\geq 60$ years & I.34I (0.663-2.7I4) & 0.414 & & & & \\
\hline \multicolumn{7}{|l|}{ Gender } \\
\hline Male & I & & & & & \\
\hline Female & $0.835(0.417-1.673)$ & 0.611 & & & & \\
\hline \multicolumn{7}{|l|}{ Body mass index } \\
\hline$<24 \mathrm{~kg} / \mathrm{m}^{2}$ & 1 & & & & & \\
\hline$\geq 24 \mathrm{~kg} / \mathrm{m}^{2}$ & $0.734(0.370-1.453)$ & 0.375 & & & & \\
\hline \multicolumn{7}{|l|}{ Approach } \\
\hline Laparotomy & 1 & & 1 & & 1 & \\
\hline Laparoscopic surgery & $0.237(0.09 \mid-0.613)$ & 0.003 & $0.453(0.149-1.374)$ & 0.162 & $0.490(0.169-1.416)$ & 0.188 \\
\hline \multicolumn{7}{|l|}{ Operative time } \\
\hline$<180 \min$ & I & & & & & \\
\hline$\geq 180 \min$ & $1.304(0.653-2.604)$ & 0.453 & & & & \\
\hline \multicolumn{7}{|l|}{ Tumor site } \\
\hline Gastric & I & & 1 & & I & \\
\hline Extra-gastric & 3.417 (1.735-6.729) & $<0.001$ & $1.974(0.912-4.272)$ & 0.084 & $2.927(1.417-6.045)$ & 0.004 \\
\hline \multicolumn{7}{|l|}{ Tumor rupture } \\
\hline No & 1 & & 1 & & 1 & \\
\hline Yes & $31.822(3.798-266.649)$ & 0.001 & $21.305(2.125-213.603)$ & 0.009 & $5.522(0.643-47.435)$ & 0.119 \\
\hline \multicolumn{7}{|l|}{ Tumor size } \\
\hline$<5 \mathrm{~cm}$ & 1 & & I & & 1 & \\
\hline$\geq 5 \mathrm{~cm}$ & $3.935(1.880-8.237)$ & $<0.001$ & $0.565(0.200-1.593)$ & 0.280 & $1.012(0.429-2.385)$ & 0.979 \\
\hline \multicolumn{7}{|l|}{ Mitosis } \\
\hline$<5 / 50 \mathrm{HPFs}$ & 1 & & I & & I & \\
\hline$\geq 5 / 50 \mathrm{HPFs}$ & 4.527 (2.109-9.718) & $<0.001$ & $2.737(1.095-6.84 I)$ & 0.031 & $2.661(1.110-6.380)$ & 0.028 \\
\hline \multicolumn{7}{|l|}{ Hemoglobin } \\
\hline$<125 \mathrm{~g} / \mathrm{L}$ & 1 & & & & & \\
\hline$\geq 125 \mathrm{~g} / \mathrm{L}$ & $0.601(0.306-1.182)$ & 0.140 & & & & \\
\hline \multicolumn{7}{|l|}{ NAR } \\
\hline$<0.086$ & 1 & & I & & & \\
\hline$\geq 0.086$ & $8.356(3.817-18.295)$ & $<0.001$ & $4.669(1.776-12.273)$ & 0.002 & & \\
\hline \multicolumn{7}{|l|}{ Fibrinogen } \\
\hline$<3.57 \mathrm{~g} / \mathrm{L}$ & 1 & & & & & \\
\hline$\geq 3.57 \mathrm{~g} / \mathrm{L}$ & 7.639 (3.448-16.925) & $<0.001$ & $5.015(1.993-12.619)$ & 0.001 & & \\
\hline \multicolumn{7}{|l|}{ NARFIB } \\
\hline 0 & 1 & & & & 1 & \\
\hline $\mathrm{I}-2$ & $20.272(4.856-84.623)$ & $<0.001$ & & & $14.116(3.243-61.443)$ & $<0.001$ \\
\hline \multicolumn{7}{|l|}{ Adjuvant imatinib } \\
\hline No & $\mathrm{I}$ & & & & & \\
\hline Yes & $1.012(0.438-2.339)$ & 0.977 & & & & \\
\hline
\end{tabular}

Abbreviations: $\mathrm{HR}$, hazard ratio; $\mathrm{Cl}$, confidence interval; HPFs, high-powered fields; mNIH, modified National Institutes of Health classification; NAR, neutrophil-toalbumin ratio; NARFIB, score combining the neutrophil-to-albumin ratio and fibrinogen. 
analyses based on the $\mathrm{mNIH}$ classification revealed significant relationships between poor RFS and high NARFIB scores among the cases with very low/low/intermediaterisk classifications $(\mathrm{p}<0.001)$ and among patients with highrisk classification $(\mathrm{p}<0.001)$ (Figure 3$)$.

\section{Relationships Between the NARFIB Score and Clinicopathological Parameters}

The analyses demonstrated that the NARFIB score was significantly associated to gender $(\mathrm{p}=0.023)$, surgical approach $(\mathrm{p}=0.015)$, tumor size $(\mathrm{p}<0.001)$, tumor site $(\mathrm{p}=0.034)$, $\mathrm{mNIH}$ category $(\mathrm{p}<0.001)$, mitotic index $(\mathrm{p}<0.001)$, and hemoglobin concentration $(\mathrm{p}=0.005)$. However, no significant relationships were observed with age $(\mathrm{p}=0.248)$, BMI
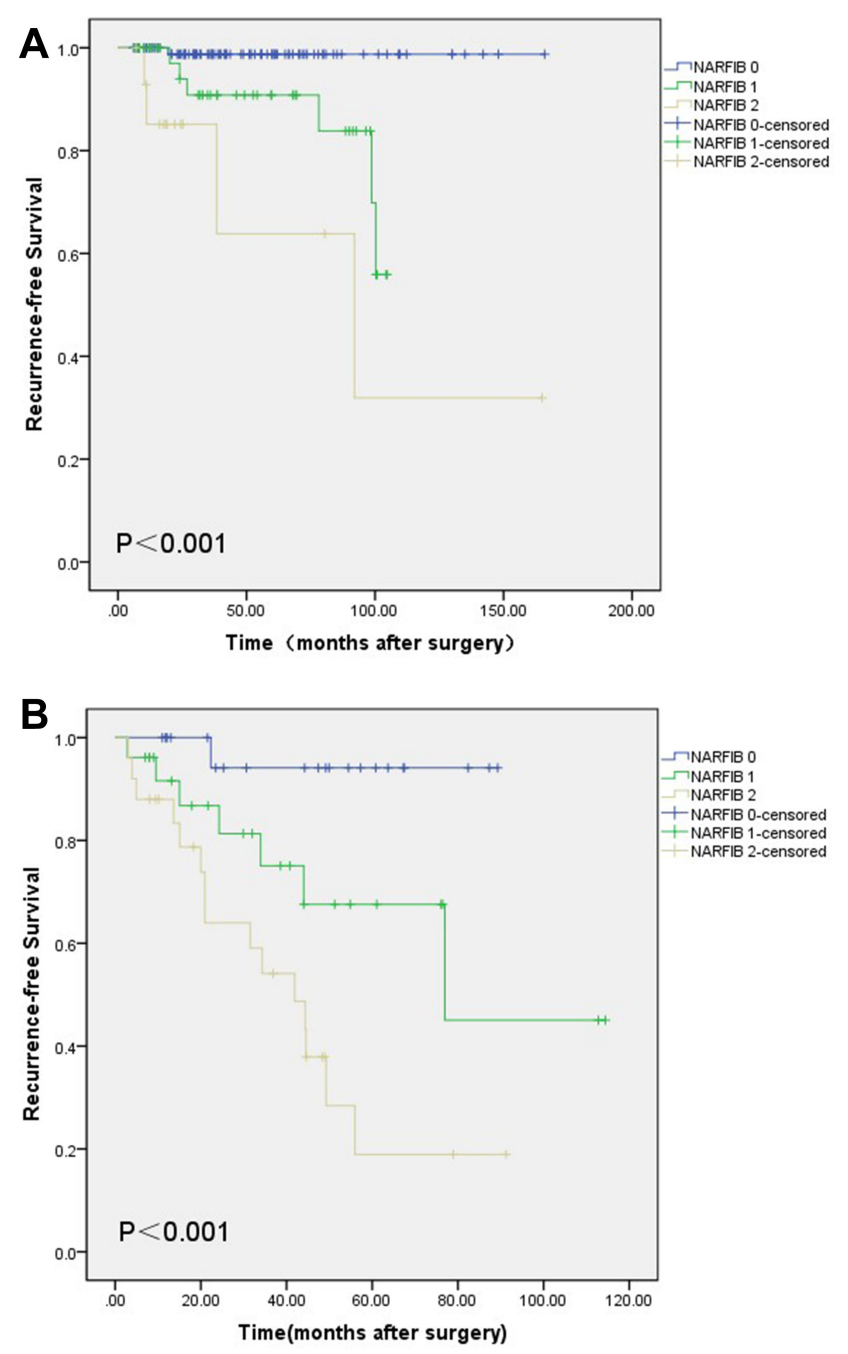

Figure 3 Prognostic value of the pretreatment NARFIB score in the different $\mathrm{mNIH}$ category subgroups. (A) The very low/low/intermediate-risk classification subgroups. (B) The high-risk classification subgroup.

Abbreviations: $\mathrm{mNIH}$, modified National Institutes of Health classification; NARFIB, score combining the neutrophil-to-albumin ratio and fibrinogen. $(\mathrm{p}=0.659)$, operative time $(\mathrm{p}=0.177)$, tumor rupture $(\mathrm{p}=0.120)$, or adjuvant imatinib $(\mathrm{p}=0.100)$ (Table 1$)$.

\section{Discussion}

The present study first indicates that the NAR can help predict the prognosis of GIST, and that the novel NARFIB score (combining the NAR and fibrinogen concentration) provided even better prognostic value than the individual factors. Based on our results, the NARFIB score had better ability to predict RFS in this setting, relative to the NAR or fibrinogen concentration alone, and was useful for risk stratification. That information might be helpful to identify cases that might benefit from personalized treatment, such as using targeted therapy. Moreover, the subgroup analyses revealed that the NARFIB score can predict RFS among cases with very low/low/medium risk classifications and even patients with a high-risk classification.

The most commonly used clinical risk classification system for GIST is the mNIH system, although it requires pathological evaluation of surgical specimens. Furthermore, the outcomes can vary substantially among patients with the same risk classification and treatment regimen, ${ }^{3,12}$ which highlights the need for an accurate and non-invasive prognostic index. Fibrinogen is an indispensable coagulation factor that can predict the prognosis of certain tumors. As an example, Lu et $\mathrm{al}^{11}$ found that elevated fibrinogen concentrations were an independent predictor of RFS among GIST patients, and $3.7 \mathrm{~g} / \mathrm{L}$ was the optimal critical point. The present study revealed a similar relationship, although the optimal critical point defined as $3.57 \mathrm{~g} / \mathrm{L}$, which suggests that large-scale multicenter studies are needed to clarify the most effective fibrinogen critical point. In this context, fibrinogen helps regulate tumor cells' proliferation and migration, as well as angiogenesis, which is thought to be related to fibrinogen up-regulating pro-inflammatory cytokines and leading to a pro-tumor microenvironment. ${ }^{13,14}$ As an extracellular matrix protein, fibrinogen can also induce the epithelial-tomesenchymal transition to promote tumor cell migration and invasion, which involves modulating the expressions of E-cadherin and vimentin. ${ }^{15,16}$ Fibrinogen also acts as a molecular bridge to facilitate adhesion between endothelial cells, platelets, and tumor cells, which contributes to metastasis. ${ }^{17}$ Moreover, an animal model of fibrinogen deficiency revealed that a microenvironment without fibrinogen can inhibit the migration of tumor cells. ${ }^{18}$

We are only aware of two studies that have investigated the relationship between NAR and tumors. Tawfik et $\mathrm{al}^{7}$ 
found that the NAR was an independent prognosticator of a complete pathological response post neoadjuvant chemotherapy for rectal cancer, while Tingle et $\mathrm{al}^{8}$ reported that the NAR was related to survival among patients receiving palliative treatment for pancreatic cancer. These results may be related to the fact that neutrophils are essential for tumor-related inflammation, which drives the generation of reactive oxygen species as well as release of proteases to promote tumorigenesis. ${ }^{19,20}$ Neutrophils can also promote angiogenesis by releasing prokineticin 2 and vascular endothelial growth factor. $^{21}$ Moreover, neutrophils can weaken the immune system by inhibiting natural killer cells, which promotes tumor cell proliferation and extravasation. 22,23

Albumin is a parameter that is commonly applied to evaluate nutritional status, as hypoalbuminemia is related to malnutrition and weakened immunity. ${ }^{24,25}$ Hypoalbuminemia is also a prognostic factor for various tumors, which may be related to a systemic inflammatory response that induces the release of tumor-related cytokines and promotes tumorigenesis. ${ }^{26-28}$ Serum albumin can also increase the number of G0/G1 cells by suppressing Rb protein phosphorylation, which inhibits tumor cell proliferation. ${ }^{28}$

There is inconsistent information regarding the prognostic role of hemoglobin concentrations in GIST. Stotz et $\mathrm{al}^{29}$ have reported that hemoglobin is an independent prognosticator for GIST patients, while Yang et $\mathrm{al}^{30}$ reported that hemoglobin was not. We performed Cox regression analysis and observed that hemoglobin concentrations were not significantly related to the prognosis of GIST patients $(\mathrm{p}=0.14)$. It is possible that these inter-study differences are related to different critical points and patient samples, which indicates that the prognostic importance of hemoglobin in GIST patients needs further verification.

This study has several limitations. First, the small retrospective single-center design may be a source of bias. Second, partial intermediate- or high-risk cases did not undergo adjuvant therapy or to complete adjuvant therapy because of adverse drug reactions or high drug costs. Thus, it is possible that we introduced selection bias by omitting some cases with intermediate- or high-risk disease

\section{Conclusion}

The present study revealed that the NARFIB score, which combines the NAR and fibrinogen concentration, was an accurate, non-invasive, and low-cost prognostic indicator for GIST patients who were undergoing radical surgery.
This scoring system may complement the current risk classification system and guide personalized treatment and postoperative follow-up.

\section{Abbreviations}

AUC, area under the curve; CI, confidence interval; NAR, neutrophil-to-albumin ratio; GIST, gastrointestinal stromal tumor; HR, hazard ratio; RFS, relapse-free survival; ROC, receiver operating characteristics; $\mathrm{mNIH}$, modified National Institutes of Health; BMI, body mass index.

\section{Ethics Approval and Consent to Participate}

All procedures performed in studies involving human participants were in accordance with the ethical standards of the institutional and/or national research committee and with the 1964 Helsinki declaration and its later amendments or comparable ethical standards. Moreover, for investigations containing human test subjects, patients' informed consent has been obtained from the participants involved before study commencement.

\section{Funding}

There is no funding to report.

\section{Disclosure}

The authors report no conflicts of interest in this work. Rui Li and Zhen Sun contributed equally to this work and should be considered co-first authors.

\section{References}

1. Joensuu H, Hohenberger P, Corless CL. Gastrointestinal stromal tumour. Lancet. 2013;382(9896):973-983. doi:10.1016/S0140-6736(13)60106-3

2. Joensuu H, Vehtari A, Riihimaki J, et al. Risk of recurrence of gastrointestinal stromal tumour after surgery: an analysis of pooled population-based cohorts. Lancet Oncol. 2012;13(3):265-274. doi:10.1016/S1470-2045(11)70299-6

3. Joensuu H. Risk stratification of patients diagnosed with gastrointestinal stromal tumor. Hum Pathol. 2008;39(10):1411-1419. doi:10.1016/ j.humpath.2008.06.025

4. Balkwill F, Mantovani A. Inflammation and cancer: back to Virchow? Lancet. 2001;357(9255):539-545.

5. Jain A, Zhang Q, Toh HC. Awakening immunity against cancer: a 2017 primer for clinicians. Chin $J$ Cancer. 2017;36(1):67. doi:10.1186/s40880-017-0233-4

6. Itami Y, Miyake M, Tatsumi Y, et al. Preoperative predictive factors focused on inflammation-, nutrition-, and muscle-status in patients with upper urinary tract urothelial carcinoma undergoing nephroureterectomy. Int J Clin Oncol. 2019;24(5):533-545. doi:10.1007/s10147-018-01381-y

7. Tawfik B, Mokdad AA, Patel PM, Li HC, Huerta S. The neutrophil to albumin ratio as a predictor of pathological complete response in rectal cancer patients following neoadjuvant chemoradiation. Anticancer Drugs. 2016;27(9):879-883. doi:10.1097/CAD.0000000000000411 
8. Tingle SJ, Severs GR, Goodfellow M, Moir JA, White SA. NARCA: a novel prognostic scoring system using neutrophil-albumin ratio and Ca19-9 to predict overall survival in palliative pancreatic cancer. J Surg Oncol. 2018;118(4):680-686. doi:10.1002/jso.25209

9. Gan W, Yi Y, Fu Y. Fibrinogen and C-reactive protein score is a prognostic index for patients with hepatocellular carcinoma undergoing curative resection: a prognostic nomogram study. $J$ Cancer. 2018;9(1):148-156. doi:10.7150/jca.22246

10. Tang L, Liu K, Wang J, Wang C, Zhao P, Liu J. High preoperative plasma fibrinogen levels are associated with distant metastases and impaired prognosis after curative resection in patients with colorectal cancer. J Surg Oncol. 2010;102(5):428-432. doi:10.1002/jso.21668

11. Lu J, Chen S, Li X, et al. Gastrointestinal stromal tumors: fibrinogen levels are associated with prognosis of patients as blood-based biomarker. Medicine (Baltimore). 2018;97(17):e0568. doi:10.1097/ MD.0000000000010568

12. Schmieder M, Henne-Bruns D, Mayer B, et al. Comparison of different risk classification systems in 558 patients with gastrointestinal stromal tumors after R0-resection. Front Pharmacol. 2016;7:504. doi:10.3389/fphar.2016.00504

13. Dolan RD, McSorley ST, Horgan PG, Laird B, McMillan DC. The role of the systemic inflammatory response in predicting outcomes in patients with advanced inoperable cancer: systematic review and meta-analysis. Crit Rev Oncol Hematol. 2017;116:134-146.

14. Ridker PM, Howard CP, Walter V. Effects of interleukin-1 $\beta$ inhibition with canakinumab on hemoglobin A1c, lipids, C-reactive protein, interleukin-6, and fibrinogen: a phase IIb randomized, placebocontrolled trial. Circulation. 2012;126(23):2739-2748. doi:10.1161/ CIRCULATIONAHA.112.122556

15. Zhang F, Wang Y, Sun P. Fibrinogen promotes malignant biological tumor behavior involving epithelial-mesenchymal transition via the p-AKT/p-mTOR pathway in esophageal squamous cell carcinoma. J Cancer Res Clin Oncol. 2017;143(12):2413-2424. doi:10.1007/ s00432-017-2493-4

16. Shu YJ, Weng H, Bao RF, et al. Clinical and prognostic significance of preoperative plasma hyperfibrinogenemia in gallbladder cancer patients following surgical resection: a retrospective and in vitro study. $B M C$ Cancer. 2014;14:566-578. doi:10.1186/1471-2407-14-566

17. Zheng S, Shen J, Jiao Y, et al. Platelets and fibrinogen facilitate each other in protecting tumor cells from natural killer cytotoxicity. Cancer Sci. 2009;100(5):859-865. doi:10.1111/j.13497006.2009.01115.x

18. Palumbo JS, Potter JM, Kaplan LS, Talmage K, Jackson DG, Degen JL. Spontaneous hematogenous and lymphatic metastasis, but not primary tumor growth or angiogenesis, is diminished in fibrinogen-deficient mice. Cancer Res. 2002;62(23):6966-6972.
19. Coffelt SB, Wellenstein MD, de Visser KE. Neutrophils in cancer: neutral no more. Nat Rev Cancer. 2016;16(7):431-446.

20. Antonio N, Bønnelykke-Behrndtz ML, Ward LC. The wound inflammatory response exacerbates growth of pre-neoplastic cells and progression to cancer. EMBO J. 2015;34(17):2219-2236. doi:10.15252/ embj. 201490147

21. Shojaei F, Singh M, Thompson JD, Ferrara N. Role of Bv8 in neutrophil-dependent angiogenesis in a transgenic model of cancer progression. Proc Natl Acad Sci U S A. 2008;105(7):2640-2645. doi:10.1073/pnas.0712185105

22. Spiegel A, Brooks MW, Houshyar S. Neutrophils suppress intraluminal NK cell-mediated tumor cell clearance and enhance extravasation of disseminated carcinoma cells. Cancer Discov. 2016;6 (6):630-649. doi:10.1158/2159-8290.CD-15-1157

23. Bodogai M, Moritoh K, Lee-Chang C. Immunosuppressive and prometastatic functions of myeloid-derived suppressive cells rely upon education from tumor-associated B cells. Cancer Res. 2015;75 (17):3456-3465. doi:10.1158/0008-5472.CAN-14-3077

24. Garcia-Martinez R, Caraceni P, Bernardi M, Gines P, Arroyo V, Jalan R. Albumin: pathophysiologic basis of its role in the treatment of cirrhosis and its complications. Hepatology. 2013;58 (5):1836-1846.

25. Barbosa-Silva MCG. Subjective and objective nutritional assessment methods: what do they really assess? Curr Opin Clin Nutr Metab Care. 2008;11(3):248-254. doi:10.1097/MCO.0b013e3282fba5d7

26. Gupta D, Lis CG. Pretreatment serum albumin as a predictor of cancer survival: a systematic review of the epidemiological literature. Nutr J. 2010;9:69. doi:10.1186/1475-2891-9-69

27. Garcia-Martinez R, Andreola F, Mehta G, et al. Immunomodulatory and antioxidant function of albumin stabilises the endothelium and improves survival in a rodent model of chronic liver failure. J Hepatol. 2015;62(4):799-806. doi:10.1016/j.jhep.2014.10.031

28. Nojiri S, Joh T. Albumin suppresses human hepatocellular carcinoma proliferation and the cell cycle. Int J Mol Sci. 2014;15(3):5163-5174. doi:10.3390/ijms15035163

29. Stotz M, Liegl-Atzwanger B, Posch F, et al. Blood-based biomarkers are associated with disease recurrence and survival in gastrointestinal stroma tumor patients after surgical resection. PLoS One. 2016;11(7): e0159448. doi:10.1371/journal.pone.0159448

30. Yang Z, Wang F, Liu S, Guan W. Comparative clinical features and short-term outcomes of gastric and small intestinal gastrointestinal stromal tumours: a retrospective study. Sci Rep. 2019;9(1):10033. doi:10.1038/s41598-019-46520-1

\section{Publish your work in this journal}

Cancer Management and Research is an international, peer-reviewed open access journal focusing on cancer research and the optimal use of preventative and integrated treatment interventions to achieve improved outcomes, enhanced survival and quality of life for the cancer patient.
The manuscript management system is completely online and includes a very quick and fair peer-review system, which is all easy to use. Visit http://www.dovepress.com/testimonials.php to read real quotes from published authors. 